\title{
Holism and Time Symmetry
}

\author{
Peter J. Lewis \\ Department of Philosophy, University of Miami, Coral Gables, Florida, USA \\ E-mail:plewis@miami.edu
}

Editors: Ken Wharton \& Ovidiu Stoica

Article history: Submitted on August 26, 2016; Accepted on November 10, 2016; Published on November 16, 2016.

( uantum mechanics is often taken to entail holism. I examine the arguments for this claim, and find that although there is no general argument from the structure of quantum mechanics to holism, there are specific arguments for holism available within the three main realist interpretations (Bohm, Ghirardi-Rimini-Weber and many-worlds). However, Evans, Price and Wharton's sideways Einstein-Podolsky-Rosen-Bell example challenges the holistic conclusion. I show how the symmetry between the sideways and standard EinsteinPodolsky-Rosen-Bell set-ups can be used to argue against holism. I evaluate the prospects for extending this insight to more general quantum systems, with a view to producing a genuinely time-symmetric hidden variable theory. I conclude that, although this extension undermines the analogy between the sideways and standard cases, quantum mechanics without holism remains a live possibility.

Quanta 2016; 5: 85-92.

\section{Introduction}

Quantum mechanics has seemed to many commentators to entail holism, the existence of properties of compound systems that cannot be reduced to properties of their parts. However, the argument is far from straightforward. In what follows, I rehearse the standard argument from en-

(c) (1) This is an open access article distributed under the terms of the Creative Commons Attribution License CC-BY-3.0, which permits unrestricted use, distribution, and reproduction in any medium, provided the original author and source are credited. tangled states, consider its defects, and then construct a more indirect argument from the three major interpretations of quantum mechanics to a holistic conclusion. I then show how an analogy between entangled states and ordinary preparation/measurement scenarios can be used to undermine even the indirect argument. The analogy suggests that quantum mechanics should be interpreted as a time-symmetric theory, in the sense that causal influences flow both from earlier in time to later and from later to earlier. However, while the analogy is suggestive, it only holds for maximally entangled states, and hence fails when the time-symmetric approach is applied to general quantum states. I argue that the failure of the analogy is no impediment to the time-symmetric interpretation of quantum mechanics, and hence that holism is not necessarily a consequence of quantum mechanics.

\section{Arguments for holism}

The canonical argument for holism appeals to entangled states of a pair of particles. For example, consider a pair of spin-1/2 particles in the singlet state $|S\rangle=\frac{1}{\sqrt{2}}\left(\left|\uparrow_{z}\right\rangle_{1}\left|\downarrow_{z}\right\rangle_{2}-\left|\downarrow_{z}\right\rangle_{1}\left|\uparrow_{z}\right\rangle_{2}\right)$, where $\left|\uparrow_{z}\right\rangle_{1}$ and $\left|\downarrow_{z}\right\rangle_{1}$ are states in which particle 1 is $z$-spin-up and $z$-spin-down respectively, and similarly for particle 2 . According to Teller [1], the property described by state $|S\rangle$ is a holistic property of the pair of particles: it describes a relation between them that cannot be reduced to their intrinsic properties. Teller's reason is that the state $|S\rangle$ cannot be factored into the product of a state of particle 1 and a state of particle 2. But as it stands this is just the beginning of an argument. Non-factorizability is a mathematical property of a mathematical object. To make the case that 
this mathematical property tells us something about the physical properties of the particles themselves, we need to know how the mathematical object $|S\rangle$ functions as a description of the physical world. And this is precisely what is up for grabs in quantum mechanics.

Bell's theorem [2] might seem to provide some hope here [3]. Bell's theorem tells us that (subject to some plausible assumptions) no ascription of properties to the individual particles can explain the observed behavior of state $|S\rangle$ on measurement. One might be tempted to claim on this basis that we have to postulate a holistic property of the pair to explain what we observe. But what does the holistic property add? Suppose we observe that particle 1 is $z$-spin-up and particle 2 is $z$-spin-down. By itself, a holistic property of the pair corresponding to state $|S\rangle$ provides no explanation for what we observe, as $|S\rangle$ is entirely symmetric between spin-up and spindown for each particle. We could try to break the symmetry by ascribing spin properties to the two particles that are revealed on measurement, but this is precisely what Bell's theorem precludes. That is, the conclusion of Bell's theorem is not that explaining the outcomes we see requires holistic properties, but that, subject to Bell's assumptions, the outcomes we see are inexplicable. Most people, Bell included, find this conclusion unacceptable. Hence Bell [4, p.20] saw his theorem as a reductio of his assumptions: one of them must be false. But then nothing follows from Bell's theorem (at least directly) about whether the properties of the individual particles are sufficient to explain the outcomes we observe.

So is there any argument that quantum mechanics entails holism? Perhaps the best hope is to look at the various realist interpretations of quantum mechanics on offer: Bohmian hidden variable theories, Ghirardi-RiminiWeber-type spontaneous collapse theories, and Everettian many-worlds theories. Each of these theories explains the outcomes of measurements on state $|S\rangle$ by violating one of Bell's assumptions. Bohm's theory and the GhirardiRimini-Weber theory violate locality, the assumption that a measurement performed on one particle cannot affect the properties of the other. Many-worlds theories violate uniqueness, the assumption that each measurement has exactly one outcome. So for none of them does Bell's theorem directly imply that the properties of the individual particles alone are insufficient to explain those outcome. But nevertheless, each of them arguably entails holism: even though Bell's theorem does not in itself require the explanation of the measurement outcomes to involve anything over and above the properties of the individual particles, in fact each of these theories does appeal to an irreducible property of the pair of particles to explain what we see. The reason is that in each case the quantum state is typically taken to be genuinely descriptive of the properties of an entity in the world, of something like a field. This field is most readily understood as an entity residing in a multi-dimensional configuration space, although it can be argued that the field can be understood in three-dimensional terms [5]. In any event, for an entangled state like $|S\rangle$, the properties of this entity include a special connection between particles 1 and 2 that is crucial to the explanation of measurement outcomes.

Let us see briefly why holism is required in the three major realist interpretations [6]. In Bohm's theory, the field described by $|S\rangle$ pushes around a pair of particles, and the positions of the particles correspond to the outcomes of our measurements. There are initial positions of the particles such that if particle 1 is not measured, particle 2 is found to be $z$-spin-up on measurement, but if particle 1 is measured, particle 2 is found to be $z$-spin down. That is, the measurement of particle 1 affects the properties of particle 2. This process is non-local, but more to the point, it constitutes a special connection between just these two particles: the measurement of particle 1 does not affect the properties of any other particle. This connection entails counterfactual conditionals such as "If the two particles were to have their spins measured in the same direction, then they would have opposite spins in that direction". These conditional relations are not reducible to the local properties of the individual particles or of the regions of space they occupy. (The conditional relations are reducible to the properties of regions of configuration space, but these regions are not local in the relevant sense.)

A similar process is at work in the Ghirardi-RiminiWeber theory. In this case the quantum state $|S\rangle$ is a complete description of the two-particle system, and there is a small chance per unit time of the state undergoing a collapse in which it becomes highly localized in the coordinates of one particle or the other. This collapse probability only becomes significant when many particles become involved, for example on measurement. When the $z$-spin of particle 1 is measured, state $|S\rangle$ collapses to (a state close to) one or other of its terms, and hence both particles acquire $z$-spin properties. Again, this process is non-local, but the key point is the special connection between particle 1 and particle 2 embodied by the entangled state. This state again supports counterfactual conditionals, and is irreducible to the local properties of the spatial regions associated with the individual particles.

The situation in the many-worlds theory is a little more subtle. As in Ghirardi-Rimini-Weber, a quantum state like $|S\rangle$ is a complete description of the system, but unlike Ghirardi-Rimini-Weber, there is no collapse mechanism. Instead, both spin outcomes occur when a spin measurement is performed, and since the terms in the state describing each outcome hardly interact after 


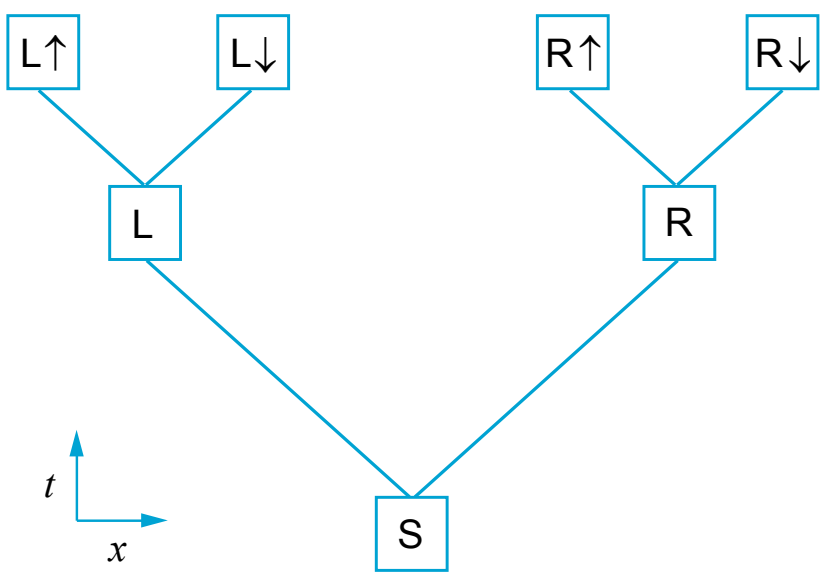

Figure 1: Spacetime diagram of the standard EinsteinPodolsky-Rosen-Bell experiment.

the measurement, they can be treated as separate worlds. When the $z$-spin of particle 1 is measured, two worlds are produced, and relative to a world, both particles acquire $z$-spin properties. There is nothing straightforwardly nonlocal in the many-worlds case, but the process via which both particles acquire spin properties still embodies a special connection between just these two particles. Again, the state supports counterfactual conditionals, relative to a world: "If the two particles were to have their spins measured in the same direction, then relative to a world, they would have opposite spins in that direction". And again, these conditional relations are not reducible to the local properties of the spatial regions associated with each particle.

So in each case, the explanation of the results we observe appeals to an irreducible property of the pair of particles (or the regions of space associated with each of them) that supports counterfactual relations between them. There are a few caveats, such as attempts by some Bohmians to regard state $|S\rangle$ as describing a law rather than a field [7]. But these aside, the entangled nature of state $|S\rangle$ genuinely corresponds to a holistic property of the ontology associated with the theory in question. So the three main realist interpretations of quantum mechanics all entail holism, and in roughly the same way. One might quite reasonably conclude that holism is an inevitable feature of the quantum world.

But such a conclusion would be premature. Taking my cue from Evans, Price and Wharton [8], I argue that a different perspective on the situation undermines the holistic conclusion.

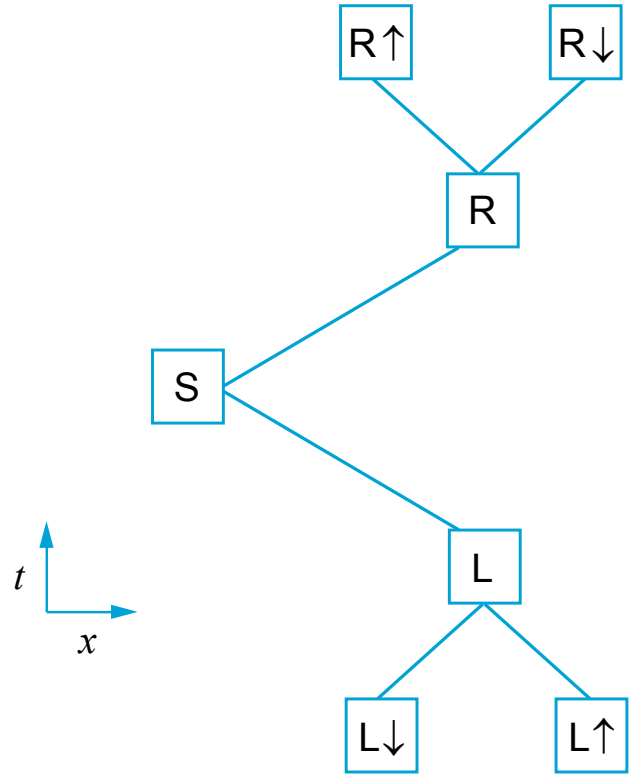

Figure 2: Spacetime diagram of the sideways EinsteinPodolsky-Rosen-Bell experiment.

\section{Looking at things sideways}

The experiment we have been considering is the standard Einstein-Podolsky-Rosen-Bell experiment. Figure 1 shows a spacetime diagram of the set-up. The particles are emitted at source $S$ and travel outwards to spin detectors at $\mathrm{L}$ and $\mathrm{R}$. The spin detectors consist of magnets that deflect particles up or down according to their spin, after which the particles may be run into a fluorescent screen which lights up at the impact point.

Figure 2, on the other hand, shows the Evans-PriceWharton sideways version of the Einstein-PodolskyRosen-Bell set-up. Here a single particle is introduced at $\mathrm{L}$ and travels to $\mathrm{R}$ via $\mathrm{S}$. $\mathrm{S}$ is in this case a device that reflects the particles, that is, that reverses their direction and their spin. $\mathrm{L}$ and $\mathrm{R}$ contain the same magnets as before: at $\mathrm{R}$ the particle is deflected up or down depending on its spin, and at $\mathrm{L}$ the particle is introduced either as a spin-up particle through the L-up channel or a spin-down particle through the L-down channel, and is deflected by the magnets onto the trajectory towards $\mathrm{S}$.

These experimental set-ups look rather different: the standard experiment involves two-particles, whereas the sideways version involves a single particle. But as Evans, Price and Wharton point out, the two experiments bear striking symmetry relations to each other. That is, if we reflect the left-hand side of Figure 1 in the $x=0$ line, and then reflect it again in the $t=0$ line, we obtain Figure 2. What is more, the probabilistic treatment of the single particle in the sideways version at two times is strongly analogous to the treatment of the two particles in the standard experiment at a single time. That is, if we 
are completely ignorant about whether the particle in the sideways version is introduced through L-up or L-down, then it is appropriate to ascribe a $50 \%$ chance to each, and consequently a $50 \%$ chance each to the particle emerging through R-up or R-down. But we know that the spin at $\mathrm{L}$ will be the opposite of the spin at R; the spins at the two times are perfectly anticorrelated. This is exactly the probability ascription appropriate for the entangled state $|S\rangle$ in the standard experiment.

But despite the analogies, there is an immediate disanalogy between the two cases: there is no temptation whatsoever to appeal to holistic properties to explain the particle's behavior in the sideways version. The overall argumentative strategy of Evans, Price and Wharton is to use the analogy between the sideways and standard experiments to dissolve the apparent problems posed by entangled states. Their target is locality: while the standard Einstein-Podolsky-Rosen-Bell experiment is often thought to require non-local action of some kind, nothing of the sort is evident in the sideways version, which casts doubt on the arguments for non-locality in the standard case. My intent is similar: while the standard EinsteinPodolsky-Rosen-Bell experiment is often thought to require holistic properties, nothing of the sort is evident in the sideways version, which casts doubt on the arguments for holism in the standard case. Let us see how that might go.

\section{Time symmetry}

Consider the sideways Einstein-Podolsky-Rosen-Bell set-up. Suppose a spin-up particle is introduced through the L-up channel. It travels via $\mathrm{S}$ to $\mathrm{R}$, where it emerges through the R-down channel. From $\mathrm{L}$ to $\mathrm{S}$ it is a $z$-spin-up particle, and from $\mathrm{S}$ to $\mathrm{R}$ it is a $z$-spin-down particle: properties ascribed to the particle at a single time suffice to explain what we observe. Similar observations apply if the particle is introduced through L-down and emerges from R-up. Of course, in the standard case, things get more problematic when the magnets at $\mathrm{L}$ and $\mathrm{R}$ can be rotated from the $z$-axis: this is the insight behind Bell's proof. But rotating the magnets causes no additional problems for the sideways version. Suppose the magnets at $\mathrm{L}$ are rotated so that they make an angle of $120^{\circ}$ with the $z$-axis; call this direction the $w$-axis. Suppose that a $w$-spin-up particle is introduced through the L-up channel. It is a $w$-spin-up particle from $\mathrm{L}$ to $\mathrm{S}$ and a $w$-spin-down particle from $\mathrm{S}$ to $\mathrm{R}$. But it is measured along the $z$-axis at $\mathrm{R}$, and we know that empirically speaking there is a $25 \%$ chance that it will be measured as $z$-spin-down and a $75 \%$ chance that it will be measured as $z$-spin-up. We could, if we like, explain this by postulating some process at $\mathrm{R}$ by which the particle acquires a $z$-spin property on measurement, for example a Ghirardi-Rimini-Weber-type collapse.

But in fact this is not required. We can simply stipulate that the $w$-spin-down particle also has a pre-existing $z$-spin property, either up or down, distributed statistically in the required 3:1 ratio. One might suspect that Bell's theorem rules out the explanation of the observed outcomes by pre-existing properties, but this is not the case because the sideways set-up explicitly violates one of Bell's assumptions, namely the independence of the particle properties from the device settings at $\mathrm{L}$ and $\mathrm{R}$ [9]. The properties of the particle clearly depend on the device setting at L: when the magnets are oriented along the $w$-axis and a particle is introduced through the L-up channel, it has to have the $w$-spin-up property if it is to be deflected towards $\mathrm{S}$ by the magnets. Given that it has this property, it is a straightforward matter to ascribe it spin-properties for any direction in which its spin might be measured at $\mathrm{R}$, without violating the predictions of quantum mechanics. If the angle between the magnets at $\mathrm{L}$ and at $\mathrm{R}$ is $\theta$, then the required statistical distribution is that the particle is spin-down in the direction of the magnets at $R$ with probability $\cos ^{2}(\theta / 2)$, and spin-up with probability $\sin ^{2}(\theta / 2)$. So in the sideways version, nothing fancy is required to explain the results we observe: the results just reflect the pre-existing properties of the particles. There is no need for holistic properties, and there is no need for a special mechanism for coordinating the spins of the two particles via a field.

Suppose we carry over this explanation to the standard Einstein-Podolsky-Rosen-Bell case. What is required is that we regard the properties of particle 1 between $S$ and $\mathrm{L}$ as dependent on the measurement that is performed at L. The twist, of course, is that in the standard case the particle between $\mathrm{S}$ and $\mathrm{L}$ is earlier in time than the measurement at $\mathrm{L}$; hence the dependence of its properties on the later measurement event is an example of backwardsin-time causation. The coherence of such accounts has been defended at length by Price [10]. The payoff is that the measurement outcomes in the standard case can be explained entirely by local particle properties that are revealed on measurement. If the magnets at $\mathrm{L}$ and $\mathrm{R}$ are both oriented along the $z$-axis, then particle 1 is either $z$-spin-up or $z$-spin-down (with a $50 \%$ probability of each), and particle 2 has the opposite $z$-spin property. If the magnets at $\mathrm{L}$ are oriented along the $w$-axis, then particle 1 is either $w$-spin-up or $w$-spin-down (with a 50\% probability of each), particle 2 has the opposite $w$-spin, and the $z$-spin property of particle 2 is statistically distributed accordingly, 3:1 in favor of $z$-spin-up if particle 2 is $w$-spin-down, and 3:1 in favor of $z$-spin-down if particle 2 is $w$-spin-up. There is no need for holism, no need 
for the non-local mechanisms of Bohm and GhirardiRimini-Weber, and no need for the world-splitting of the many-worlds theory.

One thing that is strange about the story so far, though, is that it is spatially asymmetric: the measurement performed at $\mathrm{L}$ affects the properties of the particles, but the measurement at $\mathrm{R}$ simply records them. This asymmetry reflects the obvious temporal asymmetry of the sideways case: $\mathrm{L}$ is a preparation event and $\mathrm{R}$ is a measurement event. But to inflict a spatial asymmetry on the standard case would be absurd. The obvious alternative, given the time-symmetric causation involved in the current account of correlation, is to deny both the spatial asymmetry in the standard case and the temporal asymmetry in the sideways case. That is, in the standard case particle 1 is affected by the measurement at $\mathrm{L}$, and particle 2 is affected by the measurement at $\mathrm{R}$. Indeed, because of the constraint that their total spin must be zero introduced by the manner of production of the particle pair at $S$, in fact both measurements affect the properties of both particles. And now that we are in the business of regarding causation as a time-symmetric notion, the same goes for the sideways case: the properties of the single particle are affected both by the past preparation event $\mathrm{L}$ and the future measurement event $\mathrm{R}$.

But the details introduce some complications. Consider the standard case first, and suppose that the magnets at $\mathrm{L}$ and $\mathrm{R}$ are aligned along the $z$-axis. Then it really does not matter whether we start our causal story at $\mathrm{L}$ or at $\mathrm{R}$. That is, we could say that because the magnets at $\mathrm{L}$ are along the $z$-axis, particle 1 is either $z$-spin-up or $z$-spindown (with a $50 \%$ probability of each), and because of the way the particles are produced at $\mathrm{S}$, particle 2 has the opposite spin. Alternatively, we could say that because the magnets at $\mathrm{R}$ are along the $z$-axis, particle 2 is either $z$ spin-up or $z$-spin-down (with a 50\% probability of each), and because of the way the particles are produced at $S$, particle 1 has the opposite spin. These stories are clearly compatible. But if the magnets at $\mathrm{L}$ are aligned along the $w$-axis the two stories looks different. Starting at L, we say that because of the magnet orientation, the particle is either $w$-spin-up or $w$-spin down (with a 50\% probability of each), because of the production method at $S$, particle 2 has the opposite $w$-spin, and because of particle 2's $w$-spin, its $z$-spin properties are distributed 3:1 in favor of one outcome over the other. But starting at R, we say that because of the magnet orientation, the particle is either $z$-spin-up or $z$-spin down (with a $50 \%$ probability of each), because of the production method at $S$, particle 2 has the opposite $z$-spin, and because of particle 2's $z$-spin, its $w$ spin properties are distributed 3:1 in favor of one outcome over the other. These two stories look incompatible: they seem to ascribe different probabilities to the various spin properties. For example, one story says that the $z$-spin of particle 1 is distributed evenly between up and down, and the other story says that it is distributed unevenly.

But this apparent incompatibility dissolves once we untangle what the various probabilities are conditional on. If we start at $\mathrm{L}$, then the probabilities that particle 1 is $w$-spin-up and $w$-spin down are unconditionally $50 \%$ each, and the probabilities that particle 2 is $z$-spin-up and $z$-spin down are $75 \%$ and $25 \%$ respectively, given that particle 1 is $w$-spin-up, and $25 \%$ and $75 \%$ respectively, given that particle 1 is $w$-spin down. If we start at $\mathrm{R}$, then the probabilities that particle 2 is $z$-spin-up and $z$-spin down are unconditionally $50 \%$ each, and the probabilities that particle 1 is $w$-spin-up and $w$-spin down are 75\% and $25 \%$ respectively, given that particle 1 is $z$-spin-up, and $25 \%$ and $75 \%$ respectively, given that particle 1 is $z$-spin down. These probability ascriptions are perfectly compatible, and in fact reflect what we observe in an Einstein-Podolsky-Rosen-Bell experiment. That is, if we just look at the results for particle 1 (or just those for particle 2), we see that results are evenly distributed between spin-up and spin-down whichever spin direction is measured. It is only when we compare the results for the two particles that the distinctive entanglement correlations emerge. For example, if we look that the $z$-spin results for particle 2 only in cases where particle 1 was found to be $w$-spin-up, we find that they are distributed 3:1 in favor of $z$-spin-up.

So for a given pair of settings in the standard case, the two stories (the one starting at $\mathrm{L}$ and the one starting at R) are just two ways of describing the same distribution of spin properties. And we can do the same for the sideways case. That is, although it is natural to treat $\mathrm{L}$ as a preparation event and $\mathrm{R}$ as a measurement, we can just as well do the reverse. However, we need to be a little careful, because at the human level preparation and measurement are not the same kind of thing, and there is no obvious sense in which one is the time-reverse of the other. The goal here, though, is just to show that it is possible to regard the quantum process between preparation and measurement as time-symmetric. So to that end, let us construe the preparation event so that it is closely analogous to the time-reverse of a measurement event, at least as regards the description of the quantum system. That is, just as a measurement event produces a spin-up or a spin-down outcome at random with a 50/50 statistical distribution, let us suppose that preparation involves introducing a particle at random into the spin-up or spin-down channel with a 50/50 statistical distribution.

Then supposing that the magnets at $\mathrm{L}$ are aligned along the $w$-axis and those at $\mathrm{R}$ are aligned along the $z$-axis, we can describe things like this. Starting at the earlier event L, we say that because of the magnet orientation, the particle 
at time 1 is either $w$-spin-up or $w$-spin down (with a $50 \%$ probability of each), because of the reflection method at $S$, the particle at time 2 has the opposite $w$-spin, and because of this $w$-spin, its $z$-spin properties at $\mathrm{R}$ are distributed 3:1 in favor of one outcome over the other. But starting at $\mathrm{R}$, we say that because of the magnet orientation, the particle at time 2 is either $z$-spin-up or $z$-spin down (with a $50 \%$ probability of each), because of the reflection method at $\mathrm{S}$, the particle at time 1 has the opposite $z$-spin, and because of this $z$-spin, its $w$-spin properties at $\mathrm{L}$ are distributed 3:1 in favor of one outcome over the other. These two descriptions are compatible because in each case the 50/50 probabilities are unconditional, and the $75 / 25$ probabilities are conditional on the spin at the other time.

There is something odd about the latter version of the story: it is not the kind we are used to telling. But from the current perspective, the oddity is of a purely pragmatic character that has nothing to do with the structure of the quantum world. That is, given the familiar temporal asymmetries at the macroscopic level, we often prepare a system for later measurement, but we never prepare a system for earlier measurement, so we are interested in the forward-in-time story rather than the backwardin-time story. But these familiar temporal asymmetries (presumably) have an origin in entropic asymmetry that has nothing to do with the behavior of simple quantum systems [10]. So given that backwards-in-time causation is necessary in the standard case, it seems appropriate to propose a temporal symmetry in the sideways case analogous to the spatial symmetry in the standard case.

Thinking back to the arguments in favor of holism in the previous section, what all three realist interpretations of quantum mechanics have in common is that they treat the quantum state as descriptive of the properties of a physical entity. It is here that holism takes root. And now we can see how the time-symmetric account avoids the holistic conclusion: the quantum state is treated merely as a convenient summary of an observer's knowledge of the system, as part of a recipe via which the observer can calculate the probability that the system possesses a particular property. But as far as the ontology goes, the time-symmetric account just has particles and their individual properties. The fact that the entangled state $|S\rangle$ cannot be factored into a state of particle 1 and a state of particle 2 just shows that the observer's knowledge is irreducible to knowledge of particle 1 and knowledge of particle 2, since it includes counterfactual conditionals holding between them. But there need be no property of the quantum system itself encoding those conditionals: they are enforced by the causal structures described above.

\section{Towards a theory, away from an analogy}

Section 4 shows how we can supplement the quantum state with spin properties (hidden variables) to recover the observed measurement results in standard EinsteinPodolsky-Rosen-Bell cases without recourse to holistic properties of the entire system. And since it is such cases that provide the strongest prima facie case for holism, this suggests that holism is not an inevitable consequence of quantum mechanics. But we need to be careful here: this is just a single case, albeit a widely discussed one. There is no guarantee that a general recipe for ascribing properties to systems can be constructed that recovers all the empirical predictions of quantum mechanics. That is, there is no guarantee that the time-symmetric strategy can be turned into a full-fledged hidden variable theory.

So let us consider how the property ascription of the previous section might be generalized. The recipe for ascribing probabilities to properties when the $w$-axis is selected at $\mathrm{L}$ and the $z$-axis at $\mathrm{R}$ is summarized in Table 1.

\begin{tabular}{|c|c|c|c|}
\hline Table 1 & & $\mathrm{~L}$ & \\
\hline & & $\uparrow_{w}$ & $\downarrow_{w}$ \\
\hline $\mathrm{R}$ & $\uparrow_{z}$ & $3 / 8$ & $1 / 8$ \\
\hline & $\downarrow_{z}$ & $1 / 8$ & $3 / 8$ \\
\hline
\end{tabular}

Table 1 shows the unconditional probabilities of the most fine-grained properties: that is, the top left cell shows the probability of the relevant particle (or particles in the standard case) being $w$-spin-up and $z$-spin-up. The unconditional probabilities for the two possible properties at $\mathrm{L}$ can be obtained by summing the probabilities in a column: for instance, the probability of $w$-spin-up at $\mathrm{L}$ is $3 / 8+1 / 8=1 / 2$. Similarly, the unconditional probabilities for the two possible properties at $\mathrm{R}$ can be obtained by summing the probabilities in a row. The probability of a property at $\mathrm{L}$ conditional on a particular property at $\mathrm{R}$ can be found by restricting attention to the relevant row and renormalizing. That is, the probability of spin-up on the left given spin-up on the right is $(3 / 8) /(3 / 8+1 / 8)=3 / 4$. Similarly for the other conditional probabilities.

This probabilistic recipe for ascribing properties to systems can be straightforwardly generalized in a number of ways. First, suppose the $w$-axis makes an angle $\theta$ with the $z$-axis. Then the probabilities are as in Table 2.

\begin{tabular}{|c|c|c|c|}
\hline & & $\uparrow_{w}$ & $\downarrow_{w}$ \\
\hline & $\uparrow_{z}$ & $(1 / 2) \sin ^{2}(\theta / 2)$ & $(1 / 2) \cos ^{2}(\theta / 2)$ \\
\hline & $\downarrow_{z}$ & $(1 / 2) \cos ^{2}(\theta / 2)$ & $(1 / 2) \sin ^{2}(\theta / 2)$ \\
\hline
\end{tabular}


Table 2 is analogous to a recipe suggested by Price [11] in a very similar context. Second, suppose the state, rather than being the symmetric entangled state $|S\rangle$, is some general state $|\phi\rangle$, that the measurement at $\mathrm{L}$ is of a binary observable $\hat{A}$ with two non-degenerate eigenstates $\left|A_{1}\right\rangle$ and $\left|A_{2}\right\rangle$, and that the measurement at at $\mathrm{R}$ is of a binary observable $\hat{B}$ with two non-degenerate eigenstates $\left|B_{1}\right\rangle$ and $\left|B_{2}\right\rangle$. Then the recipe for assigning probabilities to the properties $A_{1}, A_{2}, B_{1}$ and $B_{2}$ corresponding to the eigenstates is given in Table 3 .

Table 3 $\mathrm{L}$

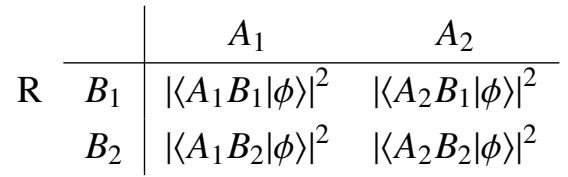

Here $\left|A_{1} B_{1}\right\rangle$ is shorthand for $\left|A_{1}\right\rangle_{1}\left|B_{1}\right\rangle_{2}$ (and so on). Finally, we can generalize further to $n$-ary observables $\hat{A}$ and $\hat{B}$ with non-degenerate eigenstates $\left|A_{i}\right\rangle$ and $\left|B_{i}\right\rangle$ (respectively). Then the recipe for ascribing probabilities to the properties $A_{i}$ and $B_{i}$ is given in Table 4.

Table 4

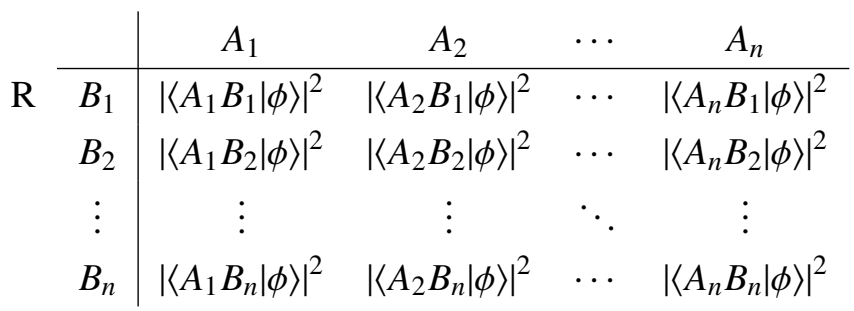

Table 4 is a fairly general recipe for assigning probabilities to properties based on the settings of the measuring devices. Note that not all potentially measurable properties of the system are assigned probabilities, but only those for which measurements are actually performed. This is what allows the time-symmetric hidden variable approach to avoid the no-go theorems of Bell [2] and Kochen-Specker [12]. Such a recipe can account for the probability distribution of measurement outcomes for a system prepared in a certain state (when $\mathrm{L}$ occurs earlier than $\mathrm{R}$, as in the sideways case), and also the measurement outcomes for entangled systems (when $L$ and $R$ are space-like separated, as in the standard case).

This looks like the beginnings of a time-symmetric approach to hidden variables. It is worth noting, though, that the analogy between sideways and standard EinsteinPodolsky-Rosen-Bell cases from which we began does not carry over to this more general context. For maximally entangled states like $|S\rangle$, a "sideways" single-particle analog exists that ascribes exactly the same probabilities to spin properties. But for partially entangled two-particle entangled states, there is no single-particle analog with the same probability ascriptions [13]. This is not a significant problem, though: while the analogy between sideways and standard cases is a useful motivating heuristic, the time-symmetric approach does not depend on any general analogy between two particles at a single time and a single particle at two times. The recipes for ascribing properties to particles in Tables 3 and 4 above still work perfectly well for entangled, partially entangled and unentangled two-particle states in standard Einstein-Podolsky-RosenBell situations, as well as for single-particle states in sideways analogs, where those analogs exist.

There is still more work to be done in developing the time-symmetric approach; there is no dynamical evolution of the state in the simple models considered here. In particular, it is not yet clear how interference should be handled in a time-symmetric hidden variable theory. There are a number of promising approaches: Price [10, pp. 252-257] suggests in general terms how a timesymmetric approach might explain interference phenomena; Spekkens [14] constructs a toy model that exhibits some of the features of interference; Sutherland [15] constructs a time-symmetric version of Bohm's theory that can generate interference effects; and Wharton [16] describes two-slit interference in time-symmetric terms, where the number of slits the particle passes through depends on the later measurement made on it. If one or more of these approaches bears fruit, then the time-symmetric approach will be a notable competitor to the interpretive approaches considered in Section 2 .

\section{Discussion}

Holism comes up repeatedly in the context of quantum mechanics, and while the route from entanglement to holism is not a clear one, a case can be made for holism, in the sense that each of the three main realist interpretations of quantum mechanics appeals to an irreducible property of both subsystems to explain the behavior of an entangled pair. But Evans, Price and Wharton's sideways perspective on entanglement is instructive here. Given the analogy between standard and sideways EinsteinPodolsky-Rosen-Bell experiments, it looks like holism should apply to both or neither. Holism is implausible in the sideways case, and the behavior of the system can be fully explained without appeal to holistic properties. So the idea is that perhaps the same kind of explanation can be applied to the standard case. And indeed it can, as long as we are prepared to accept causal influences from the future to the past. The resulting temporally symmetric account avoids the need for holistic properties, and allows the quantum state to adopt a purely epistemic role, describing the observer's knowledge of the properties of the individual particles. 
Evans, Price and Wharton do not give us a full quantum mechanical theory, just an analysis of a couple of examples; other work extends the analogy to cover all maximally entangled states, but not partially entangled states [13, 16]. However, it is straightforward to generalize the recipe for assigning probabilities to properties in the standard case to cover all states, maximally entangled or otherwise, provided one is willing to give up the analogy between the standard and sideways cases that was used to motivate the time-symmetric theory. The timesymmetric approach itself is independent of the analogy: once the approach has been recognized, the analogy can be left aside. This gives hope that a time-symmetric hidden variable theory that is provably empirically equivalent to standard quantum mechanics can be constructed. In the meantime, we can at least conclude that the path from quantum mechanics to holism is not a straightforward one.

\section{Acknowledgments}

I am grateful for the feedback from participants in the Quantum Foundations Workshop 2015 organized by Shan Gao, especially Mark Stuckey and Ken Wharton. I am also indebted to Ken Wharton for pointing out to me that the analogy between the standard and sideways cases breaks down when the entanglement is not maximal, and for very helpful comments on a draft of this paper.

\section{References}

[1] Teller P. Relational holism and quantum mechanics. British Journal for the Philosophy of Science 1986; 37(1): 71-81. JSTOR: 686998

[2] Bell JS. On the Einstein-Podolsky-Rosen Paradox. Physics 1964; 1(3): 195-200. CERN : 111654

[3] Hawthorne J, Silberstein M. For whom the Bell arguments toll. Synthese 1995; 102(1): 99-138. JSTOR: 20117977, doi : 10.1007/bf01063901

[4] Bell JS. Speakable and Unspeakable in Quantum Mechanics, 2nd edition. Cambridge: Cambridge University Press, 2004.

[5] Wallace D, Timpson CG. Quantum mechanics on spacetime I: Spacetime state realism. The British Journal for the Philosophy of Science 2010; 61(4): 697-727. PhilSci:4621, doi:10.1093/bjps/ axq010
[6] Lewis PJ. Quantum Ontology: A Guide to the Metaphysics of Quantum Mechanics. Oxford: Oxford University Press, 2016.

[7] Goldstein S, Zanghì N. Reality and the role of the wave function in quantum theory. In: The Wave Function: Essays on the Metaphysics of Quantum Mechanics. Ney A, Albert DZ (editors), Oxford: Oxford University Press, 2013, pp. 91-109 arXiv: 1101.4575

[8] Evans PW, Price H, Wharton KB. New slant on the EPR-Bell experiment. British Journal for the Philosophy of Science 2013; 64(2): 297-324. arXiv: 1001.5057, doi:10.1093/bjps/axr052

[9] Price H. A neglected route to realism about quantum mechanics. Mind 1994; 103(411): 303-336. JSTOR:2253742, arXiv:gr-qc/9406028, doi: $10.1093 / \mathrm{mind} / 103.411 .303$

[10] Price H. Time's Arrow and Archimedes' Point: New Directions for the Physics of Time. Oxford: Oxford University Press, 1996.

[11] Price H. Does time-symmetry imply retrocausality? How the quantum world says "Maybe"? Studies in History and Philosophy of Science Part B: Studies in History and Philosophy of Modern Physics 2012; 43(2): 75-83. arXiv: 1002.0906, doi : 10.1016/ j.shpsb.2011.12.003

[12] Kochen SB, Specker EP. The problem of hidden variables in quantum mechanics. Journal of Mathematics and Mechanics 1967; 17(1): 59-87. doi: 10.1512/iumj . 1968.17.17004

[13] Wharton KB, Miller DJ, Price H. Action duality: a constructive principle for quantum foundations. Symmetry 2011; 3(3): 524-540. doi:10.3390/ sym3030524

[14] Spekkens RW. Evidence for the epistemic view of quantum states: A toy theory. Physical Review A 2007; 75(3): 032110. arXiv:0401052, doi:10. 1103/PhysRevA.75.032110

[15] Sutherland RI. Causally symmetric Bohm model. Studies in History and Philosophy of Science Part B: Studies in History and Philosophy of Modern Physics 2008; 39(4): 782805. arXiv: quant-ph/0601095, doi:10.1016/ j.shpsb.2008.04.004

[16] Wharton KB. Quantum states as ordinary information. Information 2014; 5(1): 190-208. doi: 10.3390/info5010190 